\title{
Notes on generalized derivations of $*$-prime rings
}

\author{
Emine Koç and Nadeem Ur Rehman
}




\title{
NOTES ON GENERALIZED DERIVATIONS OF *-PRIME RINGS
}

\author{
EMINE KOÇ AND NADEEM UR REHMAN
}

Received 24 September, 2013

\begin{abstract}
Let $R$ be a $*$-prime ring with characteristic different from two and $U \neq 0$ be a square closed $*$-Lie ideal of $R$. An additive mapping $F: R \rightarrow R$ is called an generalized derivation if there exits a derivation $d: R \rightarrow R$ such that $F(x y)=F(x) y+x d(y)$. In the present paper, it is shown that $U \subseteq Z$ if $R$ is a $*$-prime ring which admits a generalized derivation satisfying several conditions that are associated with a derivation commuting with $*$.
\end{abstract}

2010 Mathematics Subject Classification: 16W25; 16N60; 16U80

Keywords: prime rings, derivations, generalized derivations, Lie ideals

\section{INTRODUCTION, ETC}

Let $R$ will be an associative ring with center $Z$. For any $x, y \in R$, denote the commutator $x y-y x$ by $[x, y]$ and the anti-commutator $x y+y x$ by $x \circ y$. Recall that a ring $R$ is prime if $x R y=0$ implies $x=0$ or $y=0$. An additive mapping $*: R \rightarrow R$ is called an involution if $(x y)^{*}=y^{*} x^{*}$ and $\left(x^{*}\right)^{*}=x$ for all $x, y \in R$. A ring equipped with an involution is called a ring with involution or $*$-ring. A ring with an involution is said to $*-$ prime if $x R y=x R y^{*}=0$ or $x R y=x^{*} R y=0$ implies that $x=0$ or $y=0$. Every prime ring with an involution is $*$-prime but the converse need not hold general. An example due to Oukhtite [6] justifies the above statement. Suppose that $R$ is a prime ring, $S=R \times R^{o}$ where $R^{o}$ is the opposite ring of $R$. Define involution $*$ on $S$ as $*(x, y)=(y, x)$. $S$ is $*-$ prime, but not prime. This example shows that every prime ring can be injected in a $*-$ prime ring and from this point of view $*$-prime rings constitute a more general class of prime rings. In all that follows the symbol $S_{a_{*}}(R)$, first introduced by Oukhtite, will denote the set of symmetric and skew symmetric elements of $R$, i.e. $S_{a_{*}}(R)=\left\{x \in R \mid x^{*}= \pm x\right\}$.

An additive subgroup $U$ of $R$ is said to be a Lie ideal of $R$ if $[U, R] \subseteq U$. A Lie ideal is said to be a $*$-Lie ideal if $U^{*}=U$. If $U$ is a Lie (resp. $*$-Lie) ideal of $R$, then $U$ is called a square closed Lie (resp. $*$-Lie) ideal of $R$ if $x^{2} \in U$ for all $x \in U$. 
An additive mapping $d: R \rightarrow R$ is called a derivation if $d(x y)=d(x) y+x d(y)$ holds for all $x, y \in R$. In particular, for fixed $a \in R$, the mapping $I_{a}: R \rightarrow R$ given by $I_{a}(x)=[a, x]$ is a derivation which is said to be an inner derivation. An additive function $F: R \rightarrow R$ is called a generalized inner derivation if $F(x)=a x+x b$ for fixed $a, b \in R$. For such mapping $F$, it is easy to see that

$$
F(x y)=F(x) y+x[y, b]=F(x) y+x I_{b}(y), \text { for all } x, y \in R .
$$

This observation leads to the following definition, an additive mapping $F: R \rightarrow R$ is called a generalized derivation associated with a derivation $d: R \rightarrow R$ if $F(x y)=$ $F(x) y+x d(y)$ holds for all $x, y \in R$.

Familiar examples of generalized derivations are derivations and generalized inner derivations, and the latter includes left multipliers. Since the sum of two generalized derivation is a generalized derivation, every map of the form $F(x)=c x+d(x)$, where $c$ is a fixed element of $R$ and $d$ a derivation of $R$ is a generalized derivation, and if $R$ has multiplicative identity, then all generalized derivations have this form.

Over the past thirty years, there has been an ongoing interest concerning the relationship between the commutativity of a prime ring $\mathrm{R}$ and the behavior of a special mapping on that ring. Recently, some well-known results concerning prime rings have been proved for $*-$ prime rings by Oukhtite et al. (see [4-8], where further references can be found). In the year 2005, Ashraf et al. [1] proved some commutativity theorems for prime rings. Recently the first author with Al-Omary [3] obtained the commutativity of $*$-prime ring $R$ admitting generalized derivations satisfying several conditions. Motivated by the above results, in this paper we shall discuss the situation when a $*$-prime ring $R$ which admits a generalized derivation $F$ associated with a derivation $d$ satisfying any one of the following properties: $(i) d(u) o F(v)=[u, v]$, (ii) $[d(u), F(v)]=(u \circ v)($ i ii $) d(u) F(v)=[u, v](i v) d(u) F(v)=(u \circ v)(v)$ $[d(u), F(v)]=u v$ and $(v i)(d(u) \circ F(v))=u v$.

\section{Results}

\subsection{Preliminary considerations}

The followings are some useful identities which hold for every $x, y, z \in R$. We will use them in the proof of our theorems.

- $[x, y z]=y[x, z]+[x, y] z$

- $[x y, z]=[x, z] y+x[y, z]$

- $x o(y z)=(x \circ y) z-y[x, z]=y(x \circ z)+[x, y] z$

- $(x y) \circ z=x(y \circ z)-[x, z] y=(x \circ z) y+x[y, z]$.

We begin our discussion with the following results. 
Lemma 1 ([7, Lemma 4]). Let $R$ be a $*$-prime ring with characteristic not two, $U$ be a nonzero $*$-Lie ideal of $R$ and $a, b \in R$. If $a U b=a U b^{*}=0$, then $a=0$ or $b=0$ or $U \subseteq Z$.

Lemma 2 ([2, Lemma 2.7]). Let $R$ be a $*$-prime ring with characteristic not two, $U$ be a nonzero $*-$ Lie ideal of $R$. If $a \in R$ such that $[a, U] \subseteq Z$, then either $a \in Z$ or $U \subseteq Z$.

The following Lemma is immediate consequences of Lemma 2.

Lemma 3. Let $R$ be $a *$-prime ring with characteristic not two and $U$ be a nonzero $*$-Lie ideal of $R$. Suppose that $[U, U] \subseteq Z$, then $U \subseteq Z$.

Lemma 4 ([5, Lemma 2.4]). Let $R$ be a $*$-prime ring with characteristic not two, $d$ be a nonzero derivation of $R$ which commutes with $*$ and $U$ be a nonzero $*-$ Lie ideal of $R$. If $d(U) \subseteq Z$, then $U \subseteq Z$.

Lemma 5 ([5, Lemma 2.5]). Let $R$ be a $*$-prime ring with characteristic not two, $d$ be a nonzero derivation of $R$ which commutes with $*$ and $U$ be a nonzero $*-$ Lie ideal of $R$. If $a \in R$ and $a d(U)=0(d(U) a=0)$, then $a=0$ or $U \subseteq Z$.

Lemma 6 ([5, Theorem 1.1]). Let $R$ be a $*$-prime ring with characteristic not two $d$ be a nonzero derivation of $R$ which commutes with $*$ and $U$ be a nonzero $*-$ Lie ideal of $R$. If $d^{2}(U)=0$, then $U \subseteq Z$.

\subsection{Something else}

Theorem 1. Let $R$ be $a *$-prime ring with characteristic not two and $U$ be a nonzero square closed $*-$ Lie ideal of $R$. Suppose that $R$ admits a generalized derivation $F$ associated with nonzero derivation $d$ which commutes with $*$ such that

(i) $(d(u) \circ F(v))=[u, v]$ for all $u, v \in U$, or

(ii) $[d(u), F(v)]=(u \circ v)$ for all $u, v \in U$, or

(iii) $[d(u), F(v)]=u v$ for all $u, v \in U$, or

(iv) $d(u) \circ F(v)=u v$ for all $u, v \in U$, or

If $F=0$ or $d \neq 0$, then $U \subseteq Z$.

Proof. Suppose on the contrary that $U \nsubseteq Z$. Write $L=[U, U]$, then it is easy to show that $L$ is a Lie ideal and $d(L) \subseteq U$. Moreover, since $U \nsubseteq Z$, then $L \nsubseteq Z$ by Lemma 3.

(i) If $F=0$, then by Lemma 3, we get $U \subseteq Z$, a contradiction.

Henceforth, we shall assume that $d \neq 0$. We have

$$
(d(u) \circ F(v))=[u, v] \text { for all } u, v \in U .
$$

Replacing $v$ by $2 v w$ in (2.1) and using (2.1), and the fact that $\operatorname{char} R \neq 2$, we conclude that

$$
-F(v)[d(u), w]+v(d(u) \circ d(w))+[d(u), v] d(w)=v[u, w],
$$


for all $u, v, w \in U$. Now, choose $w=d(u)$ for all $u \in L$ in the above expression we find that

$$
v\left(d(u) \circ d^{2}(u)\right)+[d(u), v] d^{2}(u)=v[u, d(u)] .
$$

Again, replace $v$ by $2 v w$ in (2.3) and use (2.3), to get

$$
[d(u), v] U d^{2}(u)=0 \text { for all } v \in U, u \in L .
$$

Since $U$ is a nonzero square closed $*$-Lie ideal of $R, L$ is a nonzero square closed $*-$ Lie ideal of $R$, too. Hence using $* d=d *$, we get

$$
[d(u), v] U\left(d^{2}(u)\right)^{*}=0 \text { for all } v \in U, u \in L \cap S_{a_{*}}(R) .
$$

Thus, by Lemma 1, we get either $[d(u), v]=0$, for all $v \in U$ or $d^{2}(u)=0$ for each $u \in L \cap S_{a_{*}}(R)$. Let $u \in L$, as $u+u^{*}, u-u^{*} \in L \cap S_{a_{*}}(R)$ and $\left[d\left(u \mp u^{*}\right), v\right]=0$, for all $v \in U$ or $d^{2}\left(u \mp u^{*}\right)=0$. Hence, we have $[d(u), v]=0$ or $d^{2}(u)=0$, for all $v \in U, u \in L$. We obtain that $L$ is the set theoretic union of two its proper subgroups viz.

$$
A=\{u \in L \mid[d(u), v]=0\}
$$

and

$$
B=\left\{u \in L \mid d^{2}(u)=0\right\} .
$$

But a group cannot be the set-theoretic union of two proper subgroups, hence $A=L$ or $B=L$. If $A=L$, then $[d(u), U]=0$ for all $u \in L$, and hence by Lemma 2 we find that $d(u) \in Z$ for all $u \in L$. Thus, by Lemma 4 and Lemma 3, we have $U \subseteq Z$, a contradiction. On the other hand, if $B=L$, then $d^{2}(L)=0$ that is, $L \subseteq Z$ by Lemma 6, and so again using Lemma 3, we get the required result.

(ii) If $F=0$, then $u \circ v=0$, for all $u, v \in U$. Replacing $v$ by $2 v w, w \in U$ in the last equation, we get $U[u, w]=0$, for all $u, w \in U$. Hence we arrive at

$$
v U[u, w]=0 \text { for all } u, v, w \in U .
$$

Since $U$ is a nonzero $*-$ Lie ideal of $R$ yields that $v^{*} U[u, w]=0$ for all $u, v, w \in U$. Hence, we have $v U[u, w]=v^{*} U[u, w]=0$ for all $u, w, v \in U$. By Lemma 1 , we get either $[u, w]=0$, for all $u, w \in U$ or $v=0$ for each $v \in U$. And so, $[u, w]=0$, for all $u, w \in U$. By Lemma 3, we obtain $U \subseteq Z$.

Therefore, we shall assume that $d \neq 0$. We have

$$
[d(u), F(v)]=u \circ v \text { for all } u, v \in U .
$$

Replacing $v$ by $2 v w$ in (2.6 and using (2.6), we conclude that

$$
F(v)[d(u), w]+v[d(u), d(w)]+[d(u), v] d(w)=-v[u, w],
$$

for all $u, v, w \in U$. For any $u \in L$, replace $w$ by $d(u)$ in (2.7), to get

$$
v\left[d(u), d^{2}(u)\right]+[d(u), v] d^{2}(u)=-v[u, d(u)],
$$


for all $v \in U, u \in L$. Now, replacing $v$ by $2 v w$ in (2.8) and using (2.8), we see that $[d(u), v] U d^{2}(u)=0$ for all $v \in U, u \in L$. Notice that the arguments given in the proof of (i) after equation (2.4) are still valid in the present situation and hence repeating the same process, we the required result.

(ii i If $F=0$, then $u v=0$, for all $u, v \in U$ and hence $u[v, w]=0$ for all $u, v, w \in U$. Now using the similar arguments as used in the proof of $(i i)$ that follows equation (2.5), we get the required result.

Therefore, we shall assume that $d \neq 0$. For any $u, v \in U$, we have

$$
[d(u), F(v)]=u v .
$$

Replacing $v$ by $2 v w$, in (2.9) and using (2.9), we arrive at

$$
F(v)[d(u), w]+[d(u), v] d(w)+v[d(u), d(w)]=0 \text { for all } u, v, w \in U .
$$

For any $u \in L$, replace $w$ by $d(u)$, we obtain that

$$
[d(u), v] d^{2}(u)+v\left[d(u), d^{2}(u)\right]=0 \text { for all } u \in L, v \in U .
$$

Now, replace $v$ by $2 v w$, in (2.10), to get

$$
2\left([d(u), v] w d^{2}(u)+v[d(u), w] d^{2}(u)+v w\left[d(u), d^{2}(u)\right]\right)=0,
$$

for all $u \in L, v, w \in U$. In the view of (2.10) the above expression yields that $2[d(u), v] w d^{2}(u)=0$, for all $u \in L, v, w \in U$. Since $\operatorname{char} R \neq 2$, we find that $[d(u), v] w d^{2}(u)=0$ for all $u \in L, v, w \in U$. that is, $[d(u), v] U d^{2}(u)=0$ for all $v \in$ $U, u \in L$, and hence using the similar arguments as used in the proof of $(i)$ that follows equation (2.4), we find the required result.

(iv) If $F=0$, then $u v=0$, for all $u, v \in U$. Using the same arguments as we used in the proof of $(i i i)$, we get the required result.

Therefore, we shall assume that $d \neq 0$. For any $u, v \in U$, we have

$$
(d(u) \circ F(v))=u v .
$$

Replacing $v$ by $2 v w$, in (2.11) and using (2.11), we obtain that

$$
-F(v)[d(u), w]+[d(u), v] d(w)+v(d(u) o d(w))=0 \text { for all } u, v, w \in U .
$$

Now, replace $w$ by $d(u)$ for all $u \in L$, to get

$$
[d(u), v] d^{2}(u)+v\left(d(u) o d^{2}(u)\right)=0 \text { for all } u \in L, v \in U .
$$

Now, applying similar technique as the one used after equation (2.10) in the proof of (ii i), we get the required result. 
Theorem 2. Let $R$ be a $*$-prime ring with characteristic not two and $U$ be a nonzero square closed $*-$ Lie ideal of $R$. Suppose that $R$ admits a generalized derivation $F$ associated with nonzero derivation $d$ which commutes with $*$ such that

(i) $d(u) F(v)=[u, v]$ for all $u, v \in U$, or

(ii) $d(u) F(v)=u \circ v$ for all $u, v \in U$.

If $F=0$ or $d \neq 0$, then $U \subseteq Z$.

Proof. ( $i$ )If $F=0$, then $[u, v]=0$ for all $u, v \in U$. Thus, by Lemma 3, we obtain that $U \subseteq Z$.

Suppose on contrary that $U \nsubseteq Z$. Therefore, we shall assume that $d \neq 0$. We have

$$
d(u) F(v)-[u, v]=0 \text { for all } u, v \in U .
$$

Replacing $u$ by $2 u w, w \in U$ in (2.12), we get

$$
2(d(u) w F(v)+u d(w) F(v)-u[w, v]-[u, v] w)=0 .
$$

Using (2.12) and $\operatorname{char} R \neq 2$, we see that

$$
d(u) w F(v)-[u, v] w=0 \text { for all } u, v, w \in U .
$$

Substituting $v$ for $u$ in (2.13), we have

$$
d(u) w F(u)=0 \text { for all } u, w \in U .
$$

That is,

$$
d(u) U F(u)=0 \text { for all } u \in U .
$$

Using $* d=d *$, we get

$$
(d(u))^{*} U F(u)=0 \text {, for all } u \in U \cap S_{a_{*}}(R) .
$$

By Lemma 1, we get either $d(u)=0$ or $F(u)=0$, for each $u \in U \cap S_{a_{*}}(R)$. Let $u \in U$, as $u+u^{*}, u-u^{*} \in U \cap S_{a_{*}}(R)$ and $d\left(u \mp u^{*}\right)=0$ or $F\left(u \mp u^{*}\right)=0$. Hence, we obtain that $d(u)=0$ or $F(u)=0$, for all $u \in U$. Let

$$
K=\{u \in U \mid d(u)=0\}
$$

and

$$
L=\{u \in U \mid F(u)=0\}
$$

of additive subgroups of $U$. Now using the same argument, we get $U=K$ or $U=L$. If $U=K$, then $U \subseteq Z$ by Lemma 4, a contradiction. If $U=L$, then $0=F(u v)=$ $F(u) v+u d(v)=u d(v)$, and so $U d(U)=(0)$. Hence $U \subseteq Z$ by Lemma 5, a contradiction. This completes the proof.

(ii) If $F=0$, then $u o v=0$, for all $u, v \in U$, and hence using the same arguments as used in the proof of Theorem 1(ii), we get $U \subseteq Z$. 
Suppose on the contrary that $U \nsubseteq Z$. Henceforth, we shall assume that $d \neq 0$. We have

$$
d(u) F(v)-u \circ v=0 \text { for all } u, v \in U .
$$

Writing $2 u w, w \in U$ by $u$ in (2.14) and using $\operatorname{char} R \neq 2$, we find that

$$
d(u) w F(v)+u d(w) F(v)-u(w o v)+[u, v] w=0 .
$$

Applying (2.14), we obtain

$$
d(u) w F(v)+[u, v] w=0 \text { for all } u, v, w \in U .
$$

Using the same arguments as used in the proof of $(i)$ after equation (2.13), we get the required result.

\section{REFERENCES}

[1] M. Ashraf, A. Ali, and R. Rani, "On generalized derivations of prime rings," Southeast Asian Bull. Math., vol. 29, no. 4, pp. 669-675, 2005.

[2] M. Ashraf and A. Khan, "Commutativity of $*$-prime rings with generalized derivations," Rend. Semin. Mat. Univ. Padova, vol. 125, pp. 71-79, 2011.

[3] Nadeem ur Rehman and R. M. Al-Omary, "On commutativity of 2-torsion free *-prime rings with generalized derivations," Mathematica, vol. 53, no. 2, pp. 171-180, 2011.

[4] L. Oukhtite and S. Salhi, "On commutativity of $\sigma$-prime rings," Glas. Mat., III. Ser., vol. 41, no. 1, pp. 57-64, 2006.

[5] L. Oukhtite and S. Salhi, "Lie ideals and derivations of $\sigma$-prime rings," Int. J. Algebra, vol. 1, no. 1-4, pp. 25-30, 2007.

[6] L. Oukhtite and S. Salhi, "On generalized derivations of $\sigma$-prime ring," Afr. Diaspora J. Math., vol. 5, no. 1, pp. 21-25, 2007.

[7] L. Oukhtite and S. Salhi, "Centralizing automorphisms and Jordan left derivations on $*$-prime rings," Adv. Algebra, vol. 1, no. 1, pp. 19-26, 2008.

[8] L. Oukhtite, S. Salhi, and L. Taoufiq, "Commutativity conditions on derivations and Lie ideals in $\sigma$-prime rings," Beitr. Algebra Geom., vol. 51, no. 1, pp. 275-282, 2010.

Authors' addresses

Emine Koç

Cumhuriyet University, Faculty of Science, Department of Mathematics, 58140, Sivas - TURKEY

E-mail address: eminekocecumhuriyet.edu.tr

\section{Nadeem Ur Rehman}

Aligarh Muslim University, Aligarh-India

E-mail address: rehman100@gmail.com 